\title{
Active Vibration Isolation Using a Dielectric Electro-Active Polymer Actuator*
}

\author{
Rahimullah SARBAN**, Richard W. JONES***, Emiliano RUSTIGHI**** \\ and Brian R. MACE**** \\ **Danfoss PolyPower A/S, Nordborg, Denmark. \\ ***University of Southern Denmark, SØnderborg, Denmark. \\ ****ISVR, University of Southampton, Southampton, U.K. \\ E-mail:brm@isvr.soton.ac.uk
}

\begin{abstract}
Dielectric Electro-Active Polymer (DEAP) devices consist of a dielectric polymer sandwiched between two electrodes. This paper describes the use of a tubular DEAP actuator for active vibration isolation. First, the quasi-static and dynamic characteristics of the actuator are discussed. These involve the voltage-strain-force behaviour of the actuator. It is seen that the actuator is inherently non-linear, involving an approximately quadratic relationship between excitation and extension under given loading conditions. In a control context, this can be compensated for either by driving the actuator about some d.c. off-set excitation in a quasi-linear manner, or by including a linearization component within the control. Next, issues concerning the frequency response over a wide range of frequency are considered. Internal resonances exist in the actuator, which limit the bandwidth over which it can be used for active control. The actuator has significant internal damping. The potential for active vibration isolation is then explored. The dynamic performance is limited by the potential bandwidth, the maximum force that can be generated and the maximum range of movement, together with the inherent nonlinearity. Performance for harmonic disturbances is investigated within an adaptive feedforward control scheme. Experimental results are presented. Good attenuation of the excitation frequency is achieved but compensation is required to get good attenuation of higher harmonics introduced by the actuator nonlinearity. Isolation in response to a band-limited random input is then demonstrated, with attenuation of $19 \mathrm{~dB}$ being achieved over a frequency range from $2-8 \mathrm{~Hz}$.
\end{abstract}

Key words: Dielectric Electro-Active Polymer, DEAP Actuator, Vibration Isolation, Feedforward Active Vibration Control

\section{Introduction}

Dielectric Electro-Active Polymer (DEAP) devices consist of a dielectric polymer sandwiched between two electrodes. They have attracted attention because they are capable of substantial changes in length when activated by electrical stimulation, have a high energy density and a relatively fast response [1,2], giving potential advantages over other actuators and materials. Consequently they can be utilised as actuators and have the potential to be an effective replacement for many conventional actuators. Of the range of 
DEAP-based actuators that have been developed, those having a cylindrical configuration are among the most promising. This paper concerns one specific design, namely a tubular actuator constructed from PolyPower ${ }^{T M}$, a silicone-based DEAP material developed by Danfoss PolyPower A/S, and its potential application to active vibration isolation. After a description of the device, in section 2 the quasi-static and dynamic characteristics of the actuator are described. The application to active vibration control in a feedforward adaptive system is then discussed, with experimental results being presented. First tonal excitation is considered, followed by the case of band-limited random noise.

\subsection{DEAP Tubular Actuator}

PolyPower $^{T M}$ is a DEAP material that is fabricated using a silicone elastomer film sandwiched between two metal electrodes. A corrugated microstructure pattern is imprinted on the elastomer and a thin metal layer spluttered on the surface, see Figure 1. The corrugation is essential to provide a degree of electrode compliance in one preferred direction. When a voltage is applied to the electrodes the force of attraction between them squeezes the nearly-incompressible soft polymer film, thereby causing an increase in area and decrease in thickness of the film and a significant change in length in the direction perpendicular to the corrugations.

The DEAP film used to construct the rolled tubular actuator considered in this paper is a laminate of two film sheets, Figure 1(b), placed back to back providing electrode corrugations on both the upper and lower surfaces of the laminate. The corrugation profile has a depth of $5 \mu \mathrm{m}$ and period of $10 \mu \mathrm{m}$, which allows for an elongation of up to $35 \%$. In addition to the active electrode-covered area, there is also a passive (non-electrode covered) area at the edges of the laminate. This avoids short circuits between the electrodes at the material edges and provides material for any mechanical connections to the actuator and the electrical connectors. The film is then rolled into a cylinder as shown in Figure 2 and end caps and electrodes attached. The fabricated tubular actuator has no internal core, is self-supporting and is stiff enough to not buckle under axial loads up to some rated value. More details about the actuator fabrication are presented in [3].

\section{Actuator characterisation}

The deformation of the actuator depends on the electrostatic pressure induced by the applied voltage $V$ and the mechanical stresses developed by the applied load $F$. These are inherently non-linear effects. Apart from the quasi-static behaviour, dynamic effects also

(a)

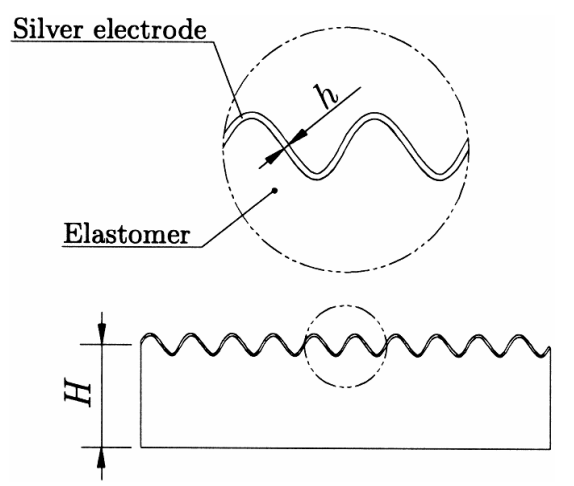

(b)

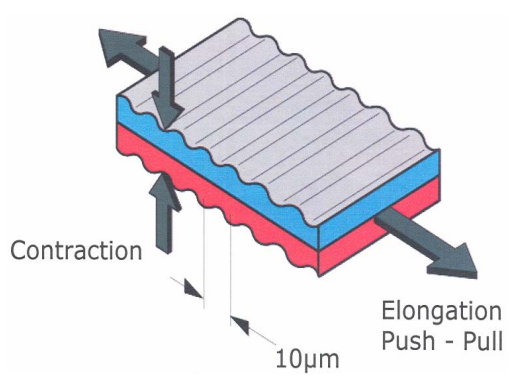

Figure 1. (a) DEAP sheet with corrugated silver electrodes. Electrode thickness, $h=100 \mathrm{~nm}$. Thickness, $H$ of the elastomer film $=40 \mu \mathrm{m}$.

(b) Schematic illustrating the elongation of a laminate of two dielectric elastomer sheets with compliant metal electrodes. 
(a)

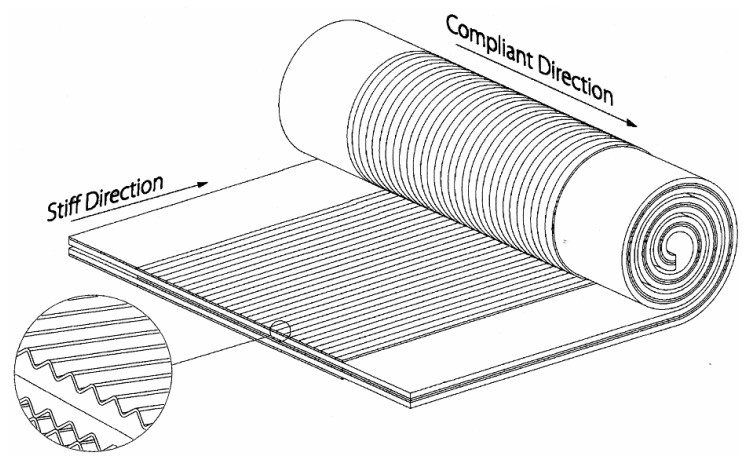

(b)

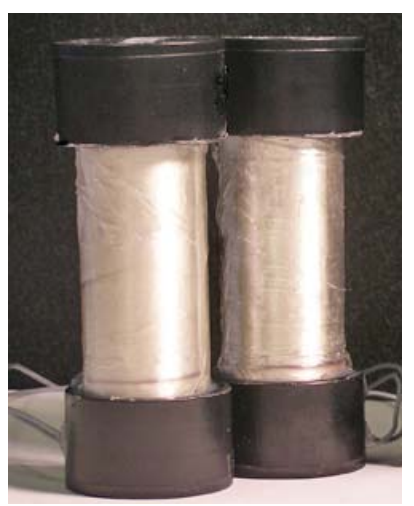

Figure 2. (a) Schematic of the tubular actuator and (b) photograph of actuators.

occur because the actuator has distributed mass and elasticity: there are internal resonances which limit the bandwidth of operation.

As far as the quasi-static characterisation is concerned, the axial mechanical stresses in the actuator are such that

$$
\sigma_{M}=\sigma_{E}+\sigma_{L}
$$

where the electrostatic stress, or Maxwell pressure, is [4]

$$
\sigma_{M}=\varepsilon_{0} \varepsilon_{r}\left(\frac{V}{t}\right)^{2}
$$

while the elastic stress $\sigma_{E}$ represents the stress due to any external loading on the actuator. It can be expressed as a modified Mooney-Rivlin equation [3].

$$
\sigma_{E}=2\left(C_{1}+C_{2}\right) h\left(\alpha^{2}-\alpha^{-2}\right)
$$

In the above, $\varepsilon_{0}$ and $\varepsilon_{r}$ are the absolute and relative dielectric constants of the DEAP material, $t$ the thickness of the laminated DEAP material, i.e. the distance between the electrodes, $C_{1}$ and $C_{2}$ are the DEAP's material elastic constants, $h$ is a hardening factor related to the thickness and material properties of the metallic electrodes and $\alpha=L / L_{0}, L$ and $L_{0}$ being the length and initial lengths of the DEAP material in the actuator. The stress caused by an applied force $F$ can be expressed as

$$
\sigma_{L}=\frac{F}{w t}
$$

where $w$ is the longitudinal length of the laminate sheet. Since the silicone-based DEAP is incompressible, its volume is conserved when a voltage is applied so that

$$
L_{0} w_{0} t_{0}=L w t
$$

Due to the corrugated metallic electrodes the width of the laminate sheet and hence the circumference of the cylinder do not change so that the thickness of the laminate becomes

$$
t=L_{0} t_{0} / L=t_{0} / \alpha
$$

From the above the voltage/load/length relation becomes 


$$
\varepsilon_{0} \varepsilon_{r}\left(\frac{V}{t_{0}}\right)^{2} \alpha^{2}=2\left(C_{1}+C_{2}\right) h\left(\alpha^{2}-\alpha^{-2}\right)+\frac{F}{w_{0} t_{0}} \alpha
$$

This can be solved numerically to find the elongation of the actuator. Note that there are nonlinearities due to the voltage-squared dependence and to nonlinear elastic effects (i.e. nonlinear dependence on $\alpha$ ). The latter are important for large-deformation applications (e.g. position control) but are less important for vibration isolation, which typically involves small amplitude motions around some equilibrium position.

Table 1 lists the parameters for the actuator used in the study and Figure 3 compares the experimental stroke/voltage data obtained under zero-load conditions and the simulated values using equation (7). There is good agreement with differences possibly due to small uncertainties in the material thickness and the hardness factor.

The dynamic characteristics of the actuator were measured by exciting it at one end and measuring the force and acceleration transmissibilities with the other end fixed or free respectively. The force transmissibility is shown in Figure 4. The actuator behaves as a uniform elastic solid with masses at each end due to the end caps. Clear resonances can be seen. The first, at about $75 \mathrm{~Hz}$, provides a limit to the bandwidth of operation. The damping is substantial, with a loss factor $\eta \approx 0.13$ which increases with frequency. This will ameliorate the effects of unwanted actuator resonances.

Table 1: Mechanical, geometric and electrical properties of the actuator.

\begin{tabular}{|c|c|}
\hline Property & Value \\
\hline Total length & $100 \mathrm{~mm}$ \\
\hline Active length & $60 \mathrm{~mm}$ \\
\hline Inner diameter & $20 \mathrm{~mm}$ \\
\hline Outer diameter & $32 \mathrm{~mm}$ \\
\hline Mass & $0.100 \mathrm{~kg}$ \\
\hline
\end{tabular}

\begin{tabular}{|c|c|}
\hline Parameter & Value \\
\hline$\varepsilon_{0}$ & $8.854 \mathrm{e}^{-12} \mathrm{~F} / \mathrm{m}$ \\
\hline$\varepsilon_{r}$ & 3.1 \\
\hline$C_{1}$ & $0.07 \mathrm{MPa}$ \\
\hline$C_{2}$ & $0.04 \mathrm{MPa}$ \\
\hline$h$ & 1.6 \\
\hline
\end{tabular}

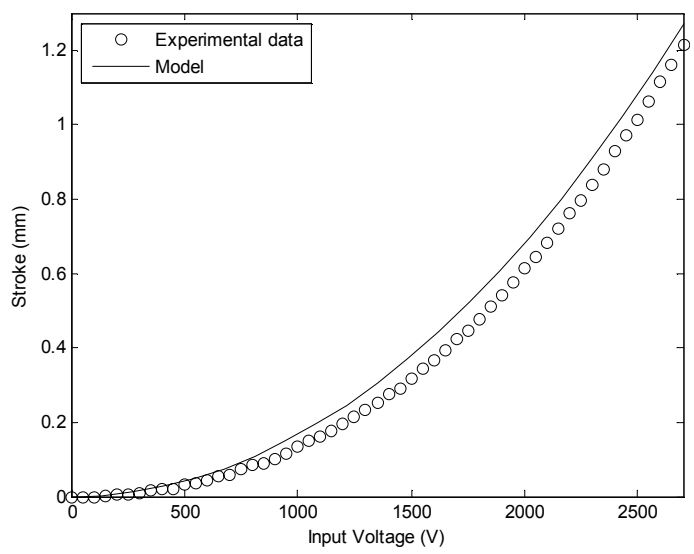

Figure 3. Simulated and measured voltage-stroke response. 

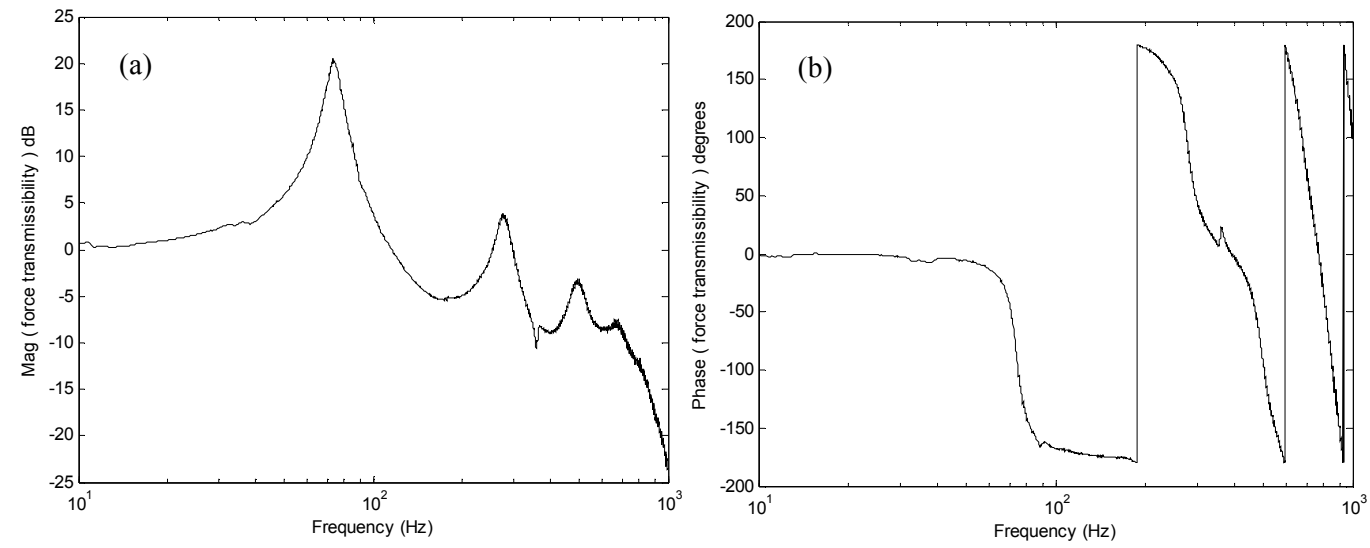

Figure 4. Force transmissibility of the tubular actuator: (a) magnitude and (b) phase.

\section{Active vibration isolation}

In this section the use of the DEAP actuator for active vibration control is investigated and experimental results are presented. The experimental set-up for tonal control is illustrated in Figure 5. It is desired to isolate a payload (here, a 250g mass) from base excitation (the disturbance from the shaker) - this involves driving the dynamic displacement of the payload to zero. The natural frequency of the mass on the actuator is $29 \mathrm{~Hz}$. The payload is supported by the DEAP actuator which is driven by some control signal. The voltage applied to the actuator $V=V_{0}+v$ comprises a d.c. voltage $V_{0}$, which gives a static displacement, and a dynamic voltage $v$.

In practice the design of such a system is subject to many constraints and compromises: the maximum voltage that can be applied to the actuator before breakdown of the dielectric occurs limits the force it can produce; actuator resonances limit the bandwidth of operation (here, to $50 \mathrm{~Hz}$ or so); the maximum stroke depends on actuator length, and the resonances are inversely proportional to this length, and so on. Moreover the actuator is nonlinear (equation (7)). In practice also the actuator might be applied in parallel with passive springs to support the static load.

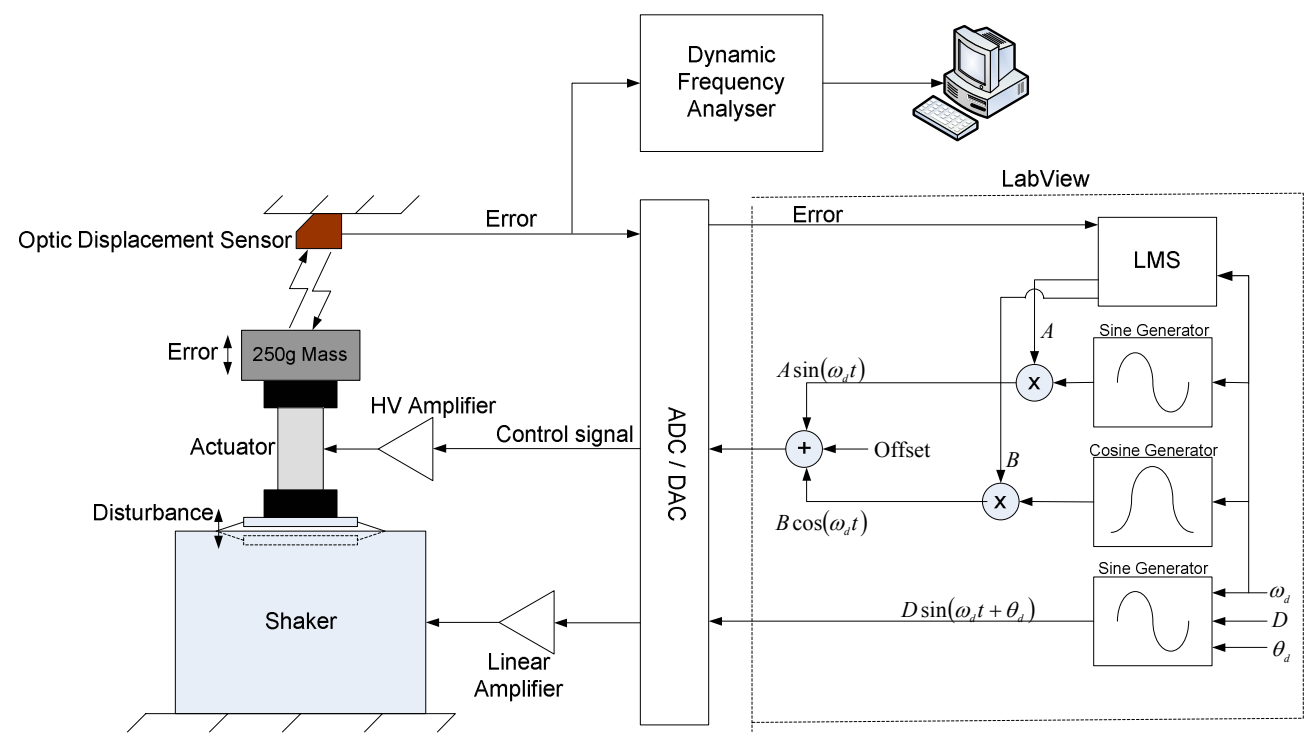

Figure 5: Schematic of the set-up for tonal active vibration isolation. 
Adaptive feedforward control was implemented using an LMS algorithm [5] and a reference signal provided by the disturbance (Figure 6). The error signal was the displacement of the payload. Two types of disturbance were considered: tonal and band-limited random noise. The feedforward controller parameters were estimated on-line and then updated using an LMS algorithm. The simplest form of this is [5]

$$
w(n+1)=w(n)-\mu r(n) e(n)
$$

where $w(n)$ is the vector of the controller parameters at time step $n$ and $r(n)$ and $e(n)$ are the reference and error signals. $\mu$ is the convergence factor which dictates the rate of convergence. The controller used depended on the form of the disturbance.

Difficulties arise because the actuator is inherently nonlinear, primarily due to the term $V^{2}$ in equation (7). If $V, \alpha$ and $F$ are written as changes from their values when the system is in static equilibrium such that

$$
V=V_{0}+v ; \quad \alpha=\alpha_{0}+\varepsilon ; \quad F=F_{0}+f
$$

the subscript 0 indicating the equilibrium value, then $\varepsilon$ and $f$ can be assumed to be small, because vibrations are typically small amplitude. Expanding equation (7) about the static equilibrium position and retaining first order terms gives

$$
\left(2 v V_{0}+v^{2}\right)=A \varepsilon+B f
$$

where $A$ and $B$ are constants. Note that $v$ need not be small compared to $V_{0}$, while the term involving $f$ can be neglected if the actuator is operating below its internal resonance. The nonlinearity remains and will introduce harmonic distortion. It can be compensated for in at least two ways. First, if $V_{0}$ is large compared to $v$ then $v^{2}<<2 v V_{0}$ and nonlinear effects are small. This involves driving the system with some substantial d.c. offset voltage and a small dynamic voltage. This in effect limits the operating range of the actuator and may hence be undesirable if the full range of actuator input voltage and stroke is to be exploited. A second approach would be to compensate directly for the nonlinearity in the controller by defining the control signal $C$ to be proportional to $\sqrt{V}$, hence linearising equation (7) to first order in $\alpha$ and $F$.

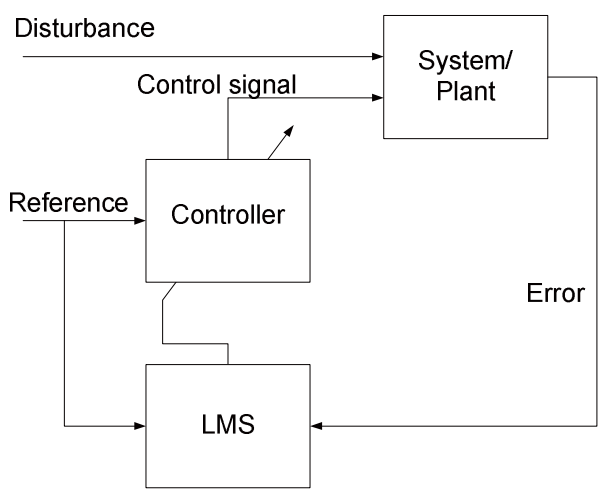

Figure 6. Block diagram of adaptive feedforward control system. 


\subsection{Harmonic disturbance}

Periodic or tonal vibration is a common excitation in reciprocating and rotating machines. Figure 5 shows a block diagram representation of the control used for a tonal disturbance. This was implemented in LabView ${ }^{\circledR}$. The d.c. offset is provided to establish some extension of the actuator at the static equilibrium position.

Two sinusoidal signals of $5 \mathrm{~Hz}$ and $10 \mathrm{~Hz}$ with different amplitudes were generated. The $5 \mathrm{~Hz}$ signal was fed to the shaker to generate the disturbance, while both signals were available as reference signals. First, a single tone controller generating $5 \mathrm{~Hz}$ sine and cosine waves of variable amplitude was used. The amplitudes of the sine and cosine waves were continuously optimized by the LMS algorithm to drive the position error of the payload to zero. (This set-up is equivalent to a FIR filter with two coefficients.)

Figure 7(a) shows the frequency response of the displacement of the payload with and without single tone control. It can be seen that the response at $5 \mathrm{~Hz}$, i.e. at the frequency of the reference signal, has been substantially reduced: the vibration has been attenuated by almost $67 \mathrm{~dB}$. However, it can also be seen that some additional background noise has been introduced over a broad range of frequencies and that the amplitude of the second and third harmonics at 10 and $15 \mathrm{~Hz}$ have been noticeably increased. This is due to the harmonic distortion introduced by the nonlinearity.

A number of tonal controllers could be used to deal with the multiple harmonics in the response. In order to attenuate both the disturbance at $5 \mathrm{~Hz}$ and the second harmonic at $10 \mathrm{~Hz}$ a two tone controller is required. The control structure is the same as the single tone controller shown in Figure 5 except there are now two targeted frequencies and two sinusoidal reference signals: at $5 \mathrm{~Hz}$ and at $10 \mathrm{~Hz}$. Two pairs of sine and cosine waves were generated at these frequencies and fed to the actuator. The amplitudes of all four signals were adapted by the LMS controller and summed before being output to the high voltage amplifier which supplies the signal to the actuator. Figure $7(\mathrm{~b})$ shows the frequency response using the two tone controller. It can be seen that the contribution of the second tone is reduced by $24 \mathrm{~dB}$ compared to the previous case.

Figure 8 shows the time response of the payload with no control, single tone control and two tone control after the controller parameters have converged. It can be seen that for single tone control the error is dominated by the residual second harmonic $10 \mathrm{~Hz}$ tone, whereas in the two tone control the effect of the $10 \mathrm{~Hz}$ tone is significantly reduced. Figure 8(b) shows the time response of the payload position after the control is switched on, showing that good convergence of the tonal controller takes place within 5 seconds or so. The convergence factor $\mu$ used in these experiments was less than half the value at which the LMS algorithm became unstable, so it is possible to further improve the rate of
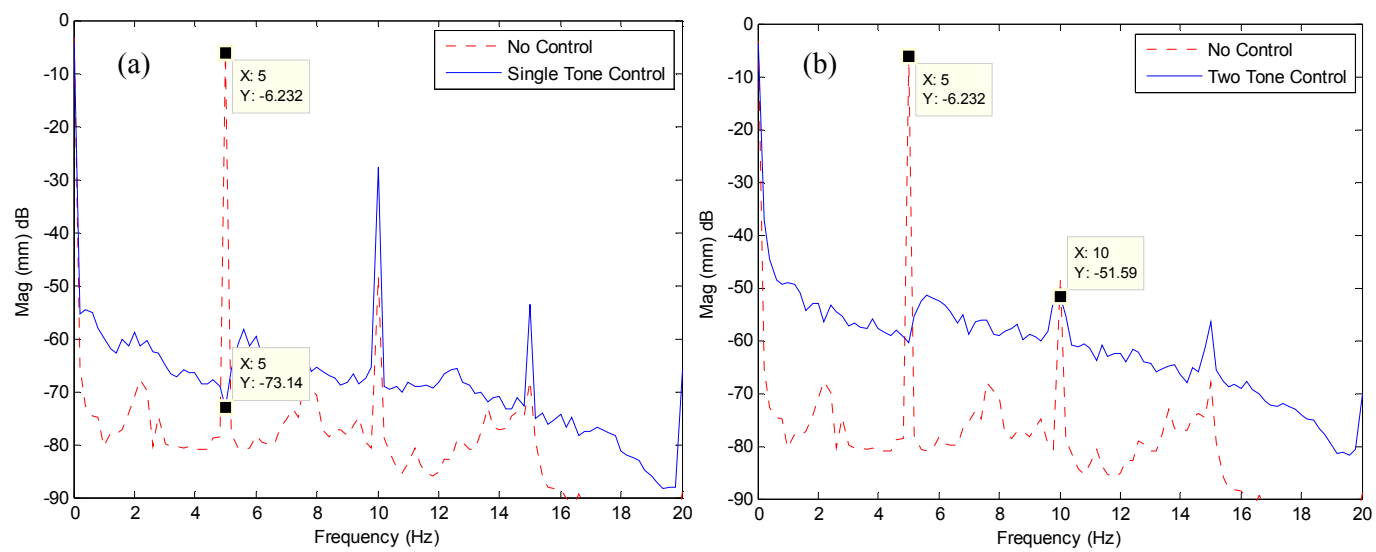

Figure 7: Control of harmonic vibrations: (a) single tone control; (b) two tone control. 

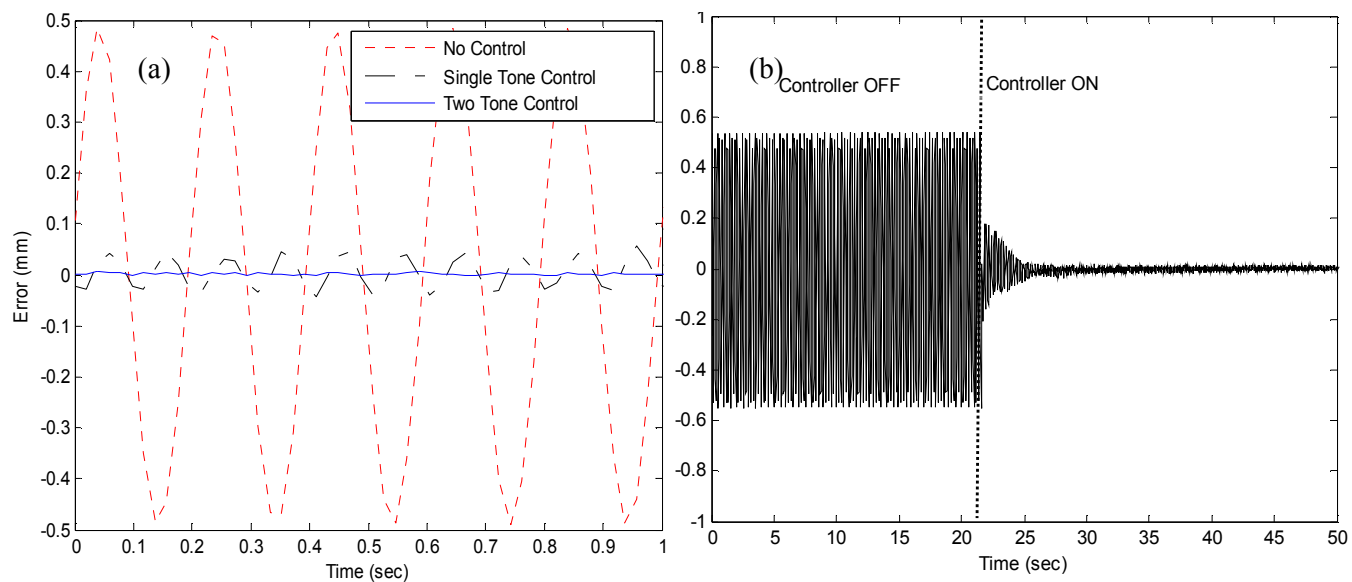

Figure 8. Time response of system: (a) steady-state response; (b) tonal controller convergence.

convergence by increasing $\mu$.

\subsection{Band-limited random disturbance}

The control structure for band-limited random excitation is shown in Figure 9. The shaker was driven by white noise band-pass filtered between 2 and $8 \mathrm{~Hz}$, this signal providing the reference signal for the LMS algorithm. Control was provided by an FIR filter, with more terms than the tonal case being required because of the richer frequency content. FIR filters of various sizes were implemented, with the number of coefficients varying up to 128 . Good control could be achieved with 16 coefficients in the FIR filter. The convergence factor was chosen to be $\mu=0.01$. Figure 10 (a) shows the frequency response of the error signal without control and after the controller had converged. Attenuation of up to $19 \mathrm{~dB}$ is obtained over the band of excitation.

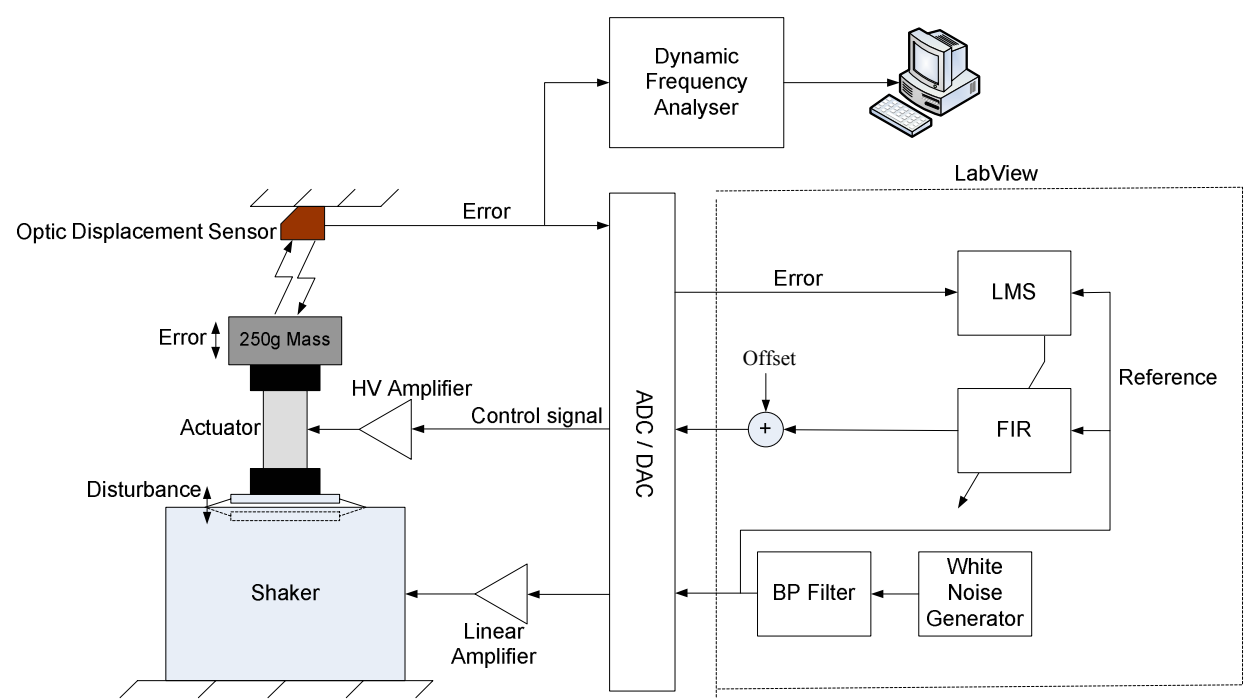

Figure 9: Schematic of the set-up for band-limited random active vibration isolation. 

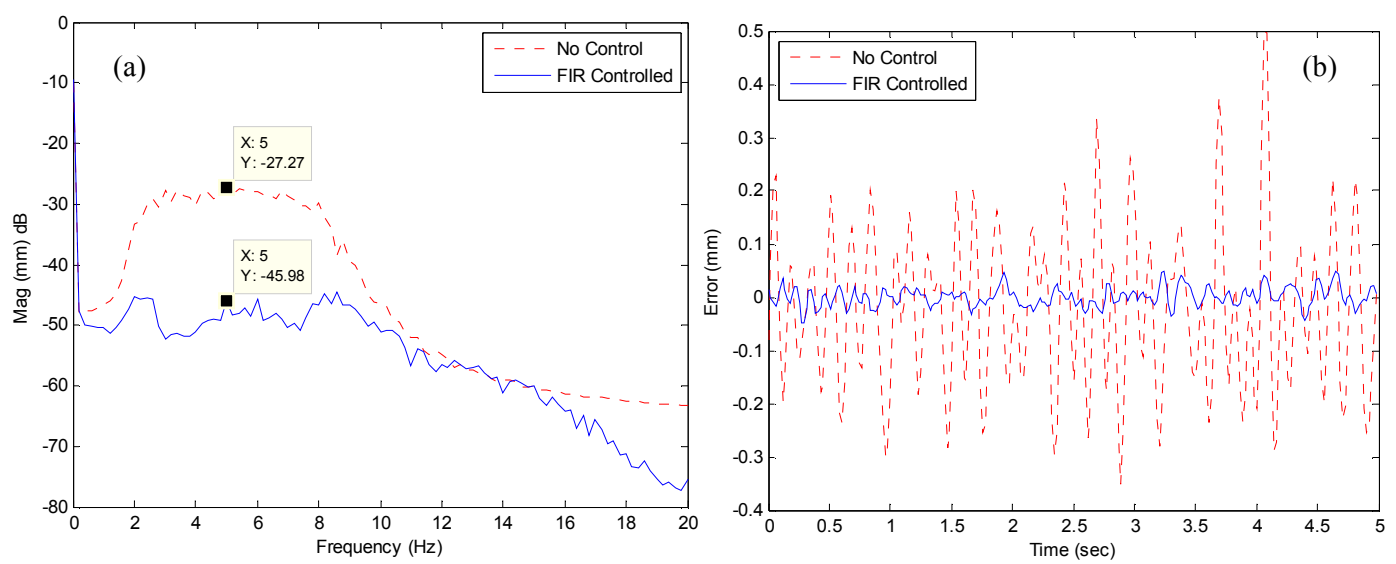

Figure 10. Band-limited vibration isolation: (a) frequency response; (b) steady-state time responses.

\section{Concluding remarks}

This paper concerned the application of a Dielectric Electro-Active Polymer (DEAP) actuator to active vibration isolation. The DEAP material consists of a dielectric polymer sandwiched between two electrodes. The material is corrugated so that it becomes compliant in one direction. It is then rolled to form a core-free tubular actuator. The actuator is capable of large displacements, up to $35 \%$ of the original length or more.

The quasi-static behaviour of the actuator (equation (7)) is nonlinear, primarily because the extension depends on the square of the applied voltage. There can also be significant nonlinear elastic effects due to the large deformations that can be generated. At high frequencies the actuator is reasonable highly damped and has internal resonances. For the device considered here the loss factor was approximately 0.13 , while there was an internal resonance at about $75 \mathrm{~Hz}$, which affects the bandwidth of operation of the device.

Experimental results were presented for the active vibration isolation of a mass from base excitation. Nonlinearities in the voltage-extension relation introduce harmonic distortion which can be reduced either by driving the actuator with a voltage that contains a significant d.c. offset or by compensating for the voltage-squared dependency in the controller. Adaptive, feedforward LMS control was implemented and two forms of disturbance were considered. For a tonal disturbance at $5 \mathrm{~Hz}$, a single-tone controller was seen to give an attenuation of nearly $67 \mathrm{~dB}$, but a significant second harmonic at $10 \mathrm{~Hz}$ was produced by the nonlinearity. Higher harmonics are also generated. Two-tone control at $5 \mathrm{~Hz}$ and $10 \mathrm{~Hz}$ simultaneously was seen to reduce the amplitude of the second harmonic by $24 \mathrm{~dB}$. A 16-coefficient FIR filter was seen to give attenuation of a band-limited random disturbance by up to $19 \mathrm{~dB}$ over the frequency band of the disturbance $(2-8 \mathrm{~Hz})$.

Future work will concentrate on examining the isolation performance of the tubular actuator for wider band random disturbances. Higher order FIR-based controllers and compensation for the nonlinearity will be required to achieve this and the possibility of using adaptive IIR filters to reduce the number of estimated parameters will be examined. In addition the use of feedback control in conjunction with adaptive feedforward control will be investigated to provide precision positioning of a sensitive system as well as vibration isolation.

\section{Acknowledgements}

The authors would like to thank Danfoss PolyPower A/S who supplied the tubular actuators. The authors would also like to acknowledge the contribution of the Danish Government for funding the Industrial PhD scholarship for Rahimullah Sarban. 


\section{References}

1. S. Ashley, Artificial Muscles, Scientific American, October, (2003), pp. 52 -59.

2. R. Pelrine, R. Kornbluh, Q. Pei, S. Stanford, S. Oh, J. Echerle, Dielectric Elastomer Artificial Muscle Actuators: Towards Biomimetric Motion, Proc. SPIE, Vol. 4695, (2002), pp. 126-137.

3. M. Tryson, H. E. Kiil and M. Benslimane, Powerful tubular core free dielectric electro activate polymer (DEAP) 'PUSH' actuator, Proceedings of SPIE, Vol 7287, (2009), pp. $72871 \mathrm{~F}-1$ to $72871 \mathrm{~F}-11$.

4. R. E. Pelrine, R. D. Kornbluh, J. P. Joseph, Electrostriction of polymer dielectrics with compliant electrodes as a mean of actuation, Sensors and Actuators A, Vol. 64, (1998), pp. $77-85$.

5. S. J. Elliot, Signal Processing for Active Control, Academic Press, London, UK (2001). 